\title{
DISTRIBUCIÓN Y CARACTERIZACIÓN DE BLOQUES ARADORES EN EL NOROESTE DE LA PENÍNSULA IBÉRICA: EL ALTO SIL Y EL MACIZO DE VIZCODI- $L L O$
}

\author{
Javier SANTOS GONZÁLEZ (javier.santos.gonzalez@uil.es) \\ Facultad de Humanidades y Ciencias Sociales. Universidad Isabel I. \\ Rosa Blanca GONZÁLEZ GUTIÉRREZ (blanca.gonzalez@unileon.es) \\ Departamento de Geografía y Geología, Universidad de León. \\ José María REDONDO VEGA (jmredv@unileon.es) \\ Departamento de Geografía y Geología, Universidad de León. \\ Amelia GÓMEZ VILLAR (agomv@unileon.es) \\ Departamento de Geografía y Geología, Universidad de León.
}

Recibido: $30 / 12 / 2015$

Aceptado: $31 / 03 / 2016$

RESUMEN: Los bloques aradores son una de las formas periglaciares más características de las montañas ibéricas, pero los estudios específicos sobre los mismos son muy escasos. En este trabajo se presentan datos de medio centenar de bloques de dos enclaves del noroeste ibérico: el Alto Sil (Cordillera Cantábrica) y el macizo de Vizcodillo (Sierra de la Cabrera). En ambos lugares se han tomado medidas de las características de los bloques (dimensiones, orientación, litología), así como de los surcos (longitud) y los montones (altura) generados, y de las pendientes (orientación, pendiente) en las que se ubican.

Se encuentran en zonas elevadas (1855-2116 m) con orientación entre norte y este, con pendientes más suaves $\left(4\right.$ a $\left.28^{\circ}\right)$ en Vizcodillo que en el Alto Sil $\left(16\right.$ y $\left.40^{\circ}\right)$, ya que en el primero de los lugares se encuentran en una superficie elevada por encima de los circos, mientras que en el Alto Sil están dentro de las laderas de los mismos. Su tamaño oscila entre 54 y los $440 \mathrm{~cm}$ de eje mayor, orientados preferentemente en el sentido de la ladera. Los surcos son relativamente cortos (menos de $1 \mathrm{~m}$ en varios casos), aunque algunos superan los 3 a $5 \mathrm{~m}$, mientras que los montones presentan una altura modesta, entre 20 y $50 \mathrm{~cm}$ en la mayoría de los casos.

Aunque la mayor parte parecen inactivos, hemos observado desplazamientos al menos en 2006 y 2015 en algunos de ellos, con movimientos de entre 10 y $35 \mathrm{~cm}$, pudiendo ser movimientos relativamente rápidos. El movimiento ocasional de los bloques parece estar ligado a la existencia de unas condiciones térmicas adecuadas durante un año concreto, si bien aún se tienen pocos datos sobre esa relación. En todo caso, en uno de los bloques se observó que la congelación estacional del suelo superaba los $50 \mathrm{~cm}$.

PALABRAS CLAVE: Periglaciarismo; Bloques aradores; Gelifluxión; Cordillera Cantábrica; Sierra de la Cabrera. 
DISTRIBUTION AND CHARACTERIZATION OF PLOUGHING BOULDERS IN NORTHWEST OF THE IBERIAN PENINSULA: THE SIL RIVER HEADWATERS AND VIZCODILLO MASSIF

ABSTRACT: Ploughing boulders are one of the most characteristic forms of periglacial environment in the Iberian Peninsula mountains, but specific studies on them are scarce. Alto Sil (Cantabrian) and solid Vizcodillo (Sierra Cabrera): In this work, fifty boulders located in two areas on northwest Spain are analyzed (Alto Sil in the Cantabrian Mountains and Vizcodillo in the Cabrera Range). In both places data about morphometric characteristics of boulders (size, orientation, lithology), furrows (length), frontal mound (height), and hillside (orientation, slope) were measured.

They are found in high altitudes (1855-2116 m) facing north to east, with gentler slopes (4 to $\left.28^{\circ}\right)$ in Vizcodillo than in the Alto Sil $\left(16\right.$ to $\left.40^{\circ}\right)$. In the first boulders are located on an elevated surface above the cirques, while in the Alto Sil are in the cirque hillsides. They are 54 to 440 $\mathrm{cm}$ long, oriented preferably in the direction of the slope. The furrows are relatively short (less than $1 \mathrm{~m}$ in many cases), although some excess of 3 to $5 \mathrm{~m}$, while the mounds has a modest height, between 20 and $50 \mathrm{~cm}$ in most cases.

Although most seem inactive, we have observed displacements at least in 2006 and 2015 in some of them. Movements of 10 to $35 \mathrm{~cm}$ were recorder. Occasional movement of the boulders seems to be linked with particular ground thermal conditions, but still they are few data on this relationship. In any case, in one of the boulders it was observed that seasonal soil freezing exceeded $50 \mathrm{~cm}$.

KEY WORDS: Periglacial processes; Ploughing boulders; Gelifluction; Cantabrian Mountains; Cabrera Range.

\section{INTRODUCCIÓN}

Los bloques aradores son una de las formas características de los ámbitos periglaciares de montaña (FRENCH, 1996), aunque también aparecen en las plataformas costeras de latitudes, con climas fríos (DiONNE, 1988). Se trata de bloques que se desplazan lentamente por una ladera provocando un montón frontal ladera abajo y un surco inmediatamente ladera arriba (WASHBURN, 1973).

Su movimiento está ligado a fenómenos de gelifluxión, en zonas de suelo con finos y congelación estacional del suelo (HREŠKO et al., 2008), que son exclusivos de áreas con permafrost o una capa helada estacional. Al avanzar, los bloques tienden a alinearse lentamente en la orientación de las laderas, siguiendo la línea de máxima pendiente (BERTHLING et al. 2001a; BALLANTYNE, 2001), aunque no faltan los bloques orientados transversalmente a la misma (CHATTOPADHYAY, 1983).

En la Península Ibérica, los bloques aradores han sido reconocidos en la mayor parte de las áreas montañosas, incluyendo el Sistema Central (FRÄNZLE, 1959; 1978), el norte del Sistema Ibérico (BROSCHE, 1978), Macizo GalaicoLeonés (BROSCHE, 1978), Cordillera Cantábrica (BROSCHE, 1978; VALCÁRCEL DÍAZ et al., 1998; Pérez Alberti et al., 1998; González Gutiérrez, 
2001; 2002; SERrano CAÑAdAS y GonZÁlez TRUEBA, 2004; SANJOSÉ BlASCO et al., 2015), Pirineos (GUTIÉRREZ ElorZA y PEÑA MonNÉ, 1981; Del BARRIO y PUIGDEFÁBREGAS, 1987; SERRANO CAÑADAS, 1995) y Sierra Nevada (OlivA et al., 2009).

Sin embargo, apenas se han realizado estudios específicos sobre estas formas, si exceptuamos las aportaciones de CARRERA GÓMEZ et al. (2005) y CARRERA GómeZ y PÉrez Alberti (2006), en la Sierra de la Cabrera, y de GARCÍA DE CELIS et al. (2006) en los Picos de Urbión.

En el presente trabajo, se analizan los bloques aradores de dos macizos montañosos del noroeste ibérico: el Alto Sil (Cordillera Cantábrica) y el macizo de Vizcodillo (Macizo Galaico-Leonés). En el primero de ellos se encuentran en torno a varias cumbres montañosas, mientras que en el segundo aparecen más concentrados en torno a la cumbre de Vizcodillo. Además de estudiar sus características morfométricas y de localización, en ambos casos se observó que algunos de los bloques presentan una dinámica actual (aunque pueda ser discontinua), lo que nos llevó a analizar qué variables podían influir en que unos estén activos y otros no.

\section{II. ÁREA DE ESTUDIO}

El área de estudio elegida es el noroeste de la Península Ibérica, dentro de la cual hemos seleccionado dos zonas donde hemos localizado diversos bloques aradores: el Alto Sil (Cordillera Cantábrica) y el Macizo de Vizcodillo (Sierra de la Cabrera, Macizo Galaico-Leonés), situados al noroeste y al suroeste de la provincia de León, respectivamente (FIGURA 1).

En el Alto Sil, los bloques aradores se encuentran repartidos por diversos circos glaciares en torno a las cumbres más elevadas, estudiándose 30 bloques en 7 localizaciones diferentes. En concreto, se encuentran en los entornos de El Miro (10 bloques), Nevadín (7), Valdeiglesia (5), Los Fueyos (3), La Brañina (2), La Losera (2) y Coronxo (1). En todos los casos se encuentran dentro de circos glaciares o formas asimilables a altitudes de entre 1.855 y $2.040 \mathrm{~m}$. Se trata de bloques en su mayoría de cuarcita ordovícica de la Serie de los Cabos, si bien también las hay precámbricas del Antiforme del Narcea (Nevadín) y un micro-conglomerado cuarcítico rosado de la Formación Cándana (La Losera). Estos bloques se encuentran dentro de zonas con formas de origen glaciar muy evidentes (SANTOS GONZÁLEZ et al., 2013), con circos bien desarrollados (GÓMEZ VILLAR et al., 2015) y en un entorno con numerosas y rotundas formas de origen periglaciar (SANTOS GONZÁLEZ, 2012), destacando los glaciares rocosos (REDONDO VEGA et al., 2010; GÓMEZ VILLAR et al., 
2011). Además, muy cerca de algunos de los bloques aradores, se desarrollan nichos de nivación activos (SANTOS GONZÁLEZ et al., 2010).

Figura 1. Localización del área de estudio, en el noroeste de la Península Ibérica. En el Alto Sil los bloques se encuentran en varias localizaciones en torno a las principales cumbres, mientras que en Vizcodillo aparecen en una sola zona.

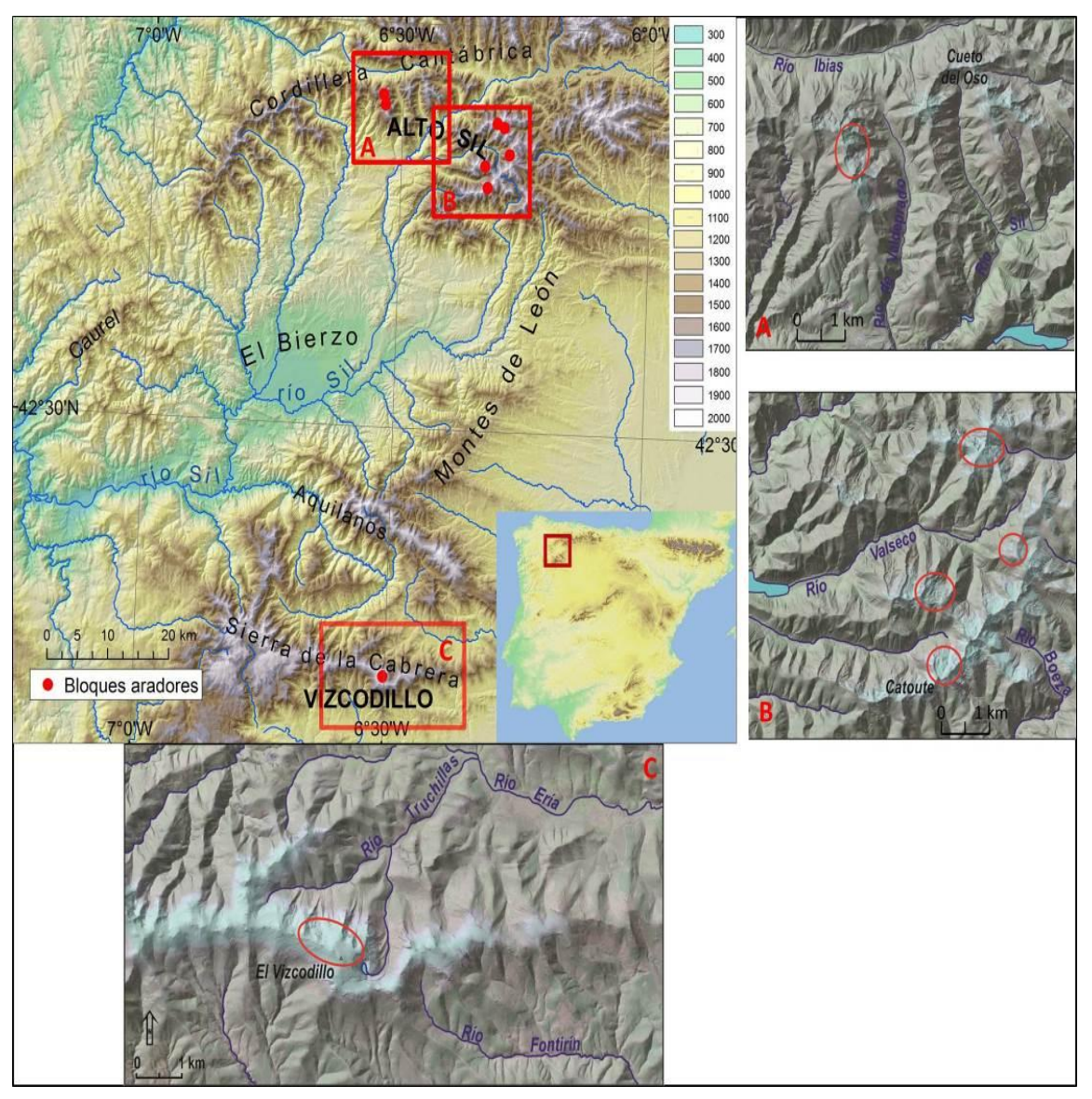

Base topográfica: IGN

FUENTE: Elaboración de los autores

En la Sierra de la Cabrera, hemos analizado 21 bloques aradores en torno a la cumbre del Vizcodillo $(2.121 \mathrm{~m})$, la segunda más elevada de ese conjunto montañoso. Se trata de bloques de cuarcita, de mayores dimensiones que los del Alto Sil, ubicados al norte y al este de la cumbre, en un suave relieve situado por encima de los circos glaciares que dieron lugar a algunas lenguas glaciares de cierta importancia (REDONDO VEGA et al. 2002). En el extremo 
oriental de esta sierra, otros bloques aradores fueron estudiados por CARRERA GÓMEZ et al. (2005) y CARRERA GÓMEZ y PÉREZ ALBERTI (2006), en condiciones similares a los analizados en este trabajo.

\section{METODOLOGÍA}

Las áreas de estudio elegidas se refieren a dos ámbitos donde los bloques aradores son relativamente comunes. Dentro de cada zona, se reconocieron mediante intenso trabajo de campo los bloques aradores que presentaran un surco y un montón frontal suficientemente desarrollados para considerarse como tales. En cada bloque, se tomaron medidas de posición (coordenadas, orientación y pendiente, tanto de la ladera en la que se ubican como del bloque), de dimensiones de los bloques (longitud, anchura, espesor, litología) y de dimensiones de los surcos (profundidad, longitud) y los montones (altura, tipo de montón) generados. Además, se observó visualmente tras la retirada de la nieve si había signos de desplazamientos recientes y el grado de recubrimiento vegetal. En caso de desplazamientos visibles, se tomaron medidas de los mismos. Por otra parte, en un bloque arador en Valdeiglesia (Alto Sil), a $2.015 \mathrm{~m}$ de altitud, se instaló un registrador de temperatura HOBO ${ }^{\circledR}$ H8 Temp, con tres sensores a 5,20 y $50 \mathrm{~cm}$ de profundidad, que registró datos entre octubre de 2002 y julio de 2007.

Los datos obtenidos sobre las medidas de los bloques aradores fueron incluidos en una tabla que ha permitido relacionar los valores obtenidos entre sí y comparar con los datos obtenidos por otros autores en trabajos dentro y fuera de la Península Ibérica. Además, se han realizado análisis de correlación, diferenciando entre aquéllos bloques que presentan evidencias de movimientos recientes y aquéllos en los que no se han observado.

\section{RESULTADOS}

\section{IV.1. Características de los bloques aradores}

Los bloques aradores están integrados por tres elementos característicos (HREŠKO et al., 2008):

- El propio bloque, que normalmente tiene al menos 40-50 $\mathrm{cm}$ de eje mayor y que tiene que estar parcialmente recubierto por suelo.

- El surco generado por el desplazamiento del bloque, que se encuentra inmediatamente ladera arriba.

— El montón frontal provocado por el movimiento del bloque, que hace que vaya acumulando material ladera abajo. Frecuentemente, los montones también pueden aparecer en los laterales del bloque. 
De los tres elementos se han tomado medidas relativas a sus dimensiones, así como la orientación de la ladera y del bloque, y la orientación y la pendiente de la ladera donde se encuentran. En total, hemos analizado 51 bloques aradores, repartidos entre el Alto Sil (30 bloques) y el macizo de Vizcodillo (21) (ANEXO 1).

La altitud a la que se encuentran varía entre 1.855 y $2.040 \mathrm{~m}$ en el Alto Sil y entre 2.000 y $2.116 \mathrm{~m}$ en Vizcodillo. En cotas más bajas, aunque aparecen bloques sueltos en algunas laderas, no hemos encontrado ninguno que presente signos de haberse desplazado por ella, por lo que parece claro que hay una altitud mínima para su desarrollo.

En cuanto a la pendiente de la ladera en la que se ubican, también se observan diferencias significativas entre el Alto Sil y Vizcodillo. Así, en el Alto Sil la pendiente oscila entre 16 y $40^{\circ}$, con un valor medio de $30,6^{\circ}$, valor muy elevado para lo habitual en este tipo de formas. Por el contrario, en Vizcodillo la pendiente media es de $11,6^{\circ}$, oscilando entre $4^{\circ}$ y $28^{\circ}$, pero solo 4 bloques se ubican en laderas de más de $15^{\circ}$. Estos valores son similares a los bloques analizados por CARRERA GÓMEZ et al. (2005) y CARRERA GÓMEZ y PÉREZ ALBERTI (2006) en la zona occidental de la sierra de la Cabrera.

El distinto valor de la pendiente está relacionado con la ubicación de los bloques. En el Alto Sil todos los bloques se encuentran dentro de la parte superior de circos glaciares o formas asimilables, por lo que la pendiente de las laderas es relativamente alta. Por el contrario, en Vizcodillo se encuentran en una plataforma por encima de los circos, donde la pendiente es más suave, y solo tres bloques están ya en el bordo de los circos, donde la pendiente aumenta.

La orientación de la ladera donde se encuentran es preferentemente norte en el Alto Sil, aunque también con bloques orientados al NE y al E, mientras que en Vizcodillo predomina la orientación E y secundariamente N y SE (FIGURA 2). En ninguna de las dos zonas hemos encontrado bloques orientados al oeste, suroeste y sur, lo cual indica una influencia clara de la orientación en su génesis, lo que, aunque la muestra no es muy numerosa, indica una influencia clara de orientación en su génesis.

Todos los bloques analizados tienen al menos $54 \mathrm{~cm}$ de eje mayor $(140 \mathrm{~cm}$ en Vizcodillo), superando el metro la mayoría. Entre las dos zonas, hay claras diferencias entre el Alto Sil y el macizo de Vizcodillo. En la primera, el valor medio de los ejes mayores es de $124 \mathrm{~cm}$, frente a los $284 \mathrm{~cm}$ de la segunda. En este último macizo varios bloques superan los $3 \mathrm{~m}$ y tres de ellos los $4 \mathrm{~m}$ de eje mayor, frente a los 2,5 m de valor máximo en el Alto Sil. Todo ello 
muestra que los bloques de Vizcodillo son de mayores dimensiones, lo que tiene que ver con el patrón de fracturación del roquedo, que hace que se generen bloques más grandes en esa zona.

Figura 2. Orientación de los bloques aradores en el Alto Sil y en Vizcodillo.

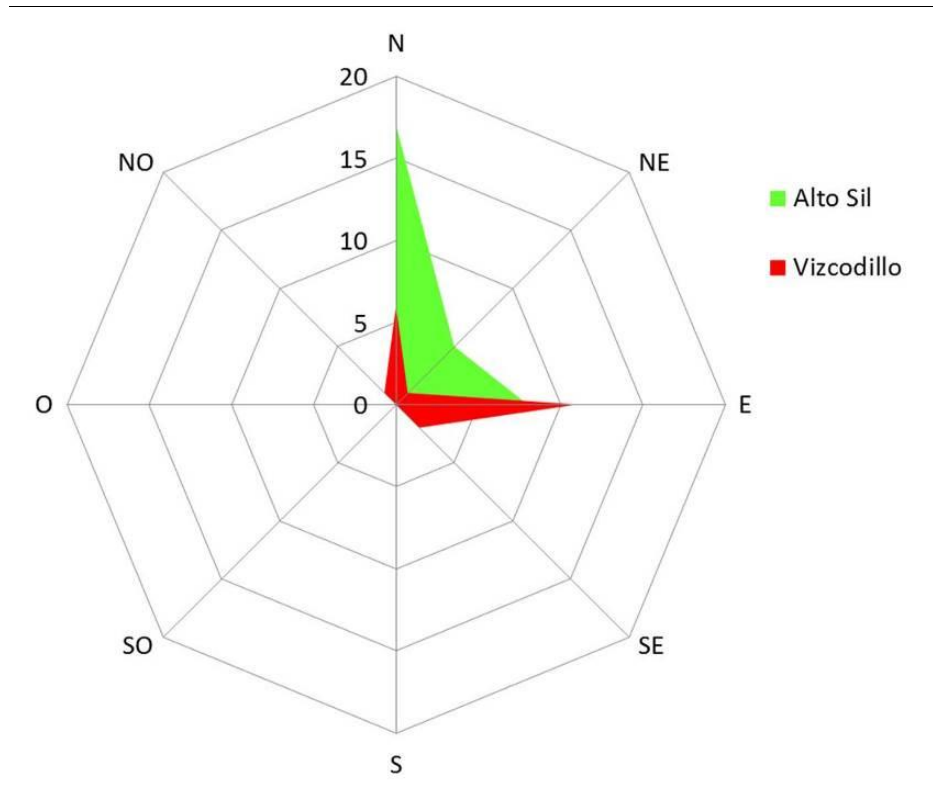

FUENTE: Elaboración de los autores

Los bloques aradores tienden a alinearse con el eje mayor siguiendo la pendiente máxima de la ladera, buscando la mínima resistencia al movimiento (BALLANTYNE, 2001), como así se ha observado en diferentes trabajos sobre estas formas (GORBUNOV, 1991; WILSON, 1993; CARRERA GÓMEZ y PÉREZ ALBERTI, 2006). Sin embargo, solo el 50\% de los bloques del Alto Sil y el $62 \%$ de los de Vizcodillo tienen una alineación que difiera menos de $20^{\circ}$ respecto a la de la ladera. La situación cambia si tenemos en cuenta solo los bloques alargados ( $\mathrm{L}>1,5 \mathrm{~A})$, puesto que en ellos la diferencia media entre la orientación es de $23^{\circ}\left(17^{\circ}\right.$ en los que tienen relación $\left.\mathrm{L} / \mathrm{A}>2\right)$, frente a $32^{\circ}$ en los que no son alargados. Es decir, los bloques más alargados se orientan mejor con respecto a la ladera que aquéllos que tienen formas más cuadrangulares.

En cuanto a los surcos, éstos están mucho mejor desarrollados en Vizcodillo (longitud media de $259 \mathrm{~cm}$ ), que en el Alto Sil (longitud media de $89 \mathrm{~cm}$ ). Así, la gran mayoría de los de Vizcodillo supera el metro de longitud y más 
de la mitad los 2 metros, mientras que en el Alto Sil menos de un tercio supera $1 \mathrm{~m}$. Esto indica que los de Vizcodillo han tenido un mayor recorrido. Estos valores están muy por debajo de los encontrados en Tien Shan (hasta $37 \mathrm{~m}$, GORBUNOV, 1991) o en Inglaterra (hasta 25 m, TUFNELL, 1972), si bien hay que tener en cuenta que la colonización vegetal y de sedimentos ha podido ocultar parte de los surcos, que dejan de ser visibles después de unos años. Así, salvo los que han tenido movimiento actual, los surcos están cubiertos por brezo y arándano, en ocasiones muy densamente.

Por su parte, la altura de los montones es bastante pequeña $(23 \mathrm{~cm}$ de media en el Alto Sil y $38 \mathrm{~cm}$ en Vizcodillo), superando los $50 \mathrm{~cm}$ solo en cuatro casos. También es reducida la cantidad de sedimentos movilizados por los bloques. En torno al $60 \%$ de los bloques tienen solo un montón frontal, el resto tienen montones fronto-laterales, en ocasiones con desarrollo en solo uno de los lados del bloque. Por último, en tres casos no se ha desarrollado montón frontal, sino solo lateral, al despegarse el bloque de la ladera con el desplazamiento.

\section{IV.2. El movimiento de los bloques}

Los bloques aradores se desplazan lentamente ladera abajo con carácter estacional. En algunas zonas de la Península Ibérica, como los Pirineos (DEL BARRIO y PUIGDEFÁBREGAS, 1987) o los Picos de Urbión (GARCÍA DE CELIS et al., 2006) se consideran formas activas, mientras que en otras, como la Cordillera Cantábrica (VALCÁRCEL DÍAZ et al., 1998; PÉREZ ALBERTI et al., 1998) o la Sierra de la Cabrera (Carrera Gómez et al., 2005; CARRERA GÓMEZ y PÉREZ AlBERTI, 2006) se piensa que son inactivos. Recientemente, en Picos de Europa se realizaron medidas de desplazamientos, aunque con movimientos registrados que se encuentran dentro del rango de error del aparato de medición (SANJOSÉ BLASCO et al., 2015).

En el caso de nuestra área de estudio, el intenso recubrimiento vegetal que presentan la mayoría de ellos en principio lleva a pensar en formas inactivas, pero hemos observado desplazamientos actuales en varios casos, limitados a años concretos, como 2006 o 2015. Los movimientos quedan en evidencia por la presencia de surcos recientes (FIGURA 3) en los que queda al descubierto el suelo contrastando con el resto de zonas cubiertas de vegetación. También se puede ver una movilización de sedimentos en la parte frontal del bloque.

En el Alto Sil, donde los bloques fueron observados durante cuatro años, sólo en uno de los años (2006) se apreciaron desplazamientos en 7 de los 30 bloques observados. En Vizcodillo, el desplazamiento de tres bloques era evidente en el año 2015, mientras que los otros 18 no presentaban ningún singo 
de movimiento actual. En cualquier caso, hay que tener en cuenta que la colonización vegetal es muy rápida en estos entornos (FIGURA 4), por lo que es posible que algunos de ellos se hayan movido, al menos algún año, en las dos últimas décadas.

Figura 3. Evidencias de desplazamientos actuales en el montón frontal (izquierda) $y$ en el surco (derecha) de bloques aradores en el entorno de Valdeiglesia (Alto Sil).
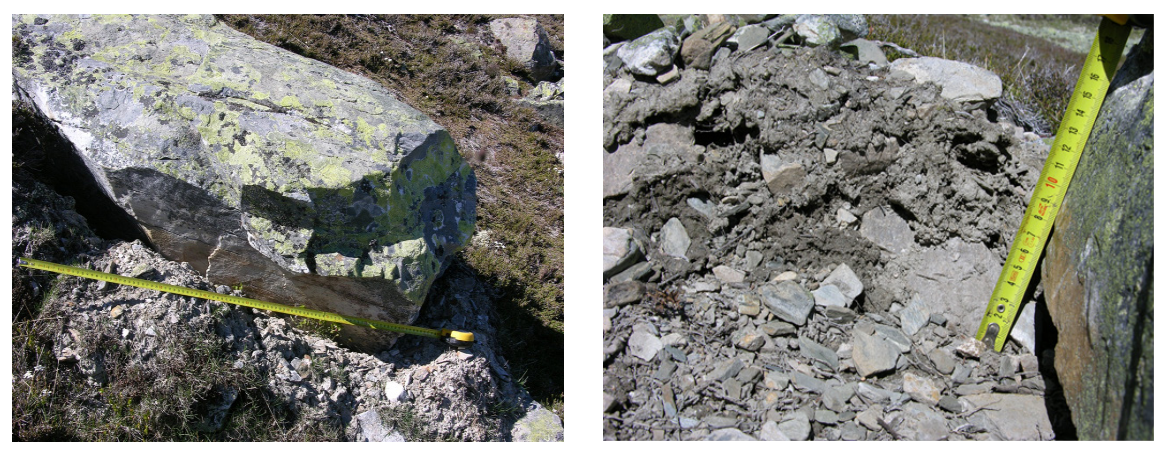

FUENTE: Elaboración de los autores

Figura 4. Aumento del recubrimiento vegetal en un bloque arador en Vizcodillo entre los años 2002 (izquierda) y 2015 (derecha)

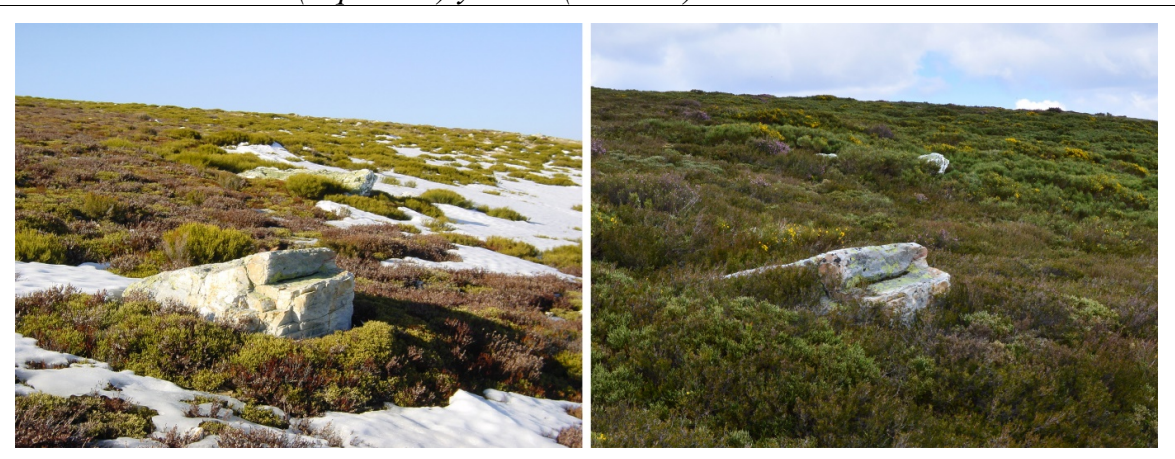

FUENTE: Fotografía de los autores

\section{IV.3. Relaciones entre variables}

Las variables analizadas han sido incluidas en una tabla de correlaciones (CUADRO 1). Aunque las relaciones entre variables no son muy significativas (salvo las que tienen que ver con las dimensiones de los bloques), sí se observan algunas de cierta transcendencia. En especial, destaca el hecho de que muestren una cierta correlación la longitud del surco con la altura del bloque 
y, correlación negativa, con la pendiente de la ladera. Es decir, los surcos tienden a estar más desarrollados en aquellos bloques que tienen más altura (lo que puede provocar surcos más profundos y que se pueden conservar durante más tiempo), y en las laderas de menor pendiente, donde la velocidad de relleno puede ser más baja.

Cuadro 1. Correlación entre las variables analizadas de los bloques aradores. Alt (altitud), L (longitud), A (anchura), a (altura), Pl (pendiente de la ladera), Ls (longitud del surco), Am (altura del montón).

\begin{tabular}{ccccc|c|c|c|c}
\hline & Alt $(\mathrm{m})$ & $L(\mathrm{~cm})$ & $A(\mathrm{~cm})$ & $a(\mathrm{~cm})$ & $P l\left({ }^{\circ}\right)$ & $L s(\mathrm{~cm})$ & $A m(\mathrm{~cm})$ \\
\hline Alt $(\mathrm{m})$ & - & & & & & & \\
$L(\mathrm{~cm})$ & 0.694 & - & & & & & \\
$A(\mathrm{~cm})$ & 0.593 & $\mathbf{0 . 8 2 6}$ & - & & & & \\
$a(\mathrm{~cm})$ & 0.603 & $\mathbf{0 . 7 0 6}$ & $\mathbf{0 , 6 9 5}$ & - & & & \\
$P l\left({ }^{\circ}\right)$ & $\mathbf{- 0 . 7 8 4}$ & $\mathbf{- 0 , 7 8 6}$ & $-0,628$ & $\mathbf{- 0 , 7 0 7}$ & - & & \\
Ls $(\mathrm{cm})$ & 0.523 & 0,618 & 0,571 & $\underline{\mathbf{0 , 6 8 7}}$ & $\overline{-}$ & - & \\
& & & & & 0,607 & & \\
Am $(\mathrm{cm})$ & 0.364 & 0,572 & 0,634 & 0,339 & - & 0,401 & - \\
& & & & & 0,384 & & \\
\hline
\end{tabular}

FUENTE: Elaboración de los autores

Si analizamos con mayor profundidad la relación existente entre algunas variables, se pueden obtener algunas conclusiones de interés.

En primer lugar, destacamos la relación existente entre la altitud y la pendiente de la ladera en la que se ubican los bloques aradores analizados (FIGURA 5). Se puede ver que los de menor altitud se encuentran en pendientes más elevadas, ya que en su mayor parte se sitúan en la parte superior de los circos glaciares. Por el contrario, los bloques inactivos de Vizcodillo están a mayor altitud y en una superficie culminante por encima de los circos, por lo que las pendientes de las laderas son menores. También se puede ver que los bloques activos, en su mayor parte, están en zonas con pendiente relativamente alta, sin que parezca tener una gran influencia la altitud.

Por otra parte, si vemos en detalle la relación existente entre la pendiente de la ladera y la longitud del surco (FIGURA 6), podemos observar que los bloques activos están instalados en laderas de fuerte pendiente y los surcos presentan longitudes más cortas (menor desplazamiento de los bloques o menor distancia recorrida) tanto en el Alto Sil como en el Vizcodillo. La situación cambia con los bloques inactivos: en el Alto Sil los bloques siguen localizándose en laderas de fuerte pendiente aunque la longitud del surco es mayor. Los del Vizcodillo aparecen claramente separados de los otros tres grupos, se localizan en pendientes mucho más suaves y los surcos son más largos (especialmente en pendientes entre 7 y $11^{\circ}$ ). 
Figura 5. Relación entre la altitud y la pendiente de la ladera de los bloques aradores

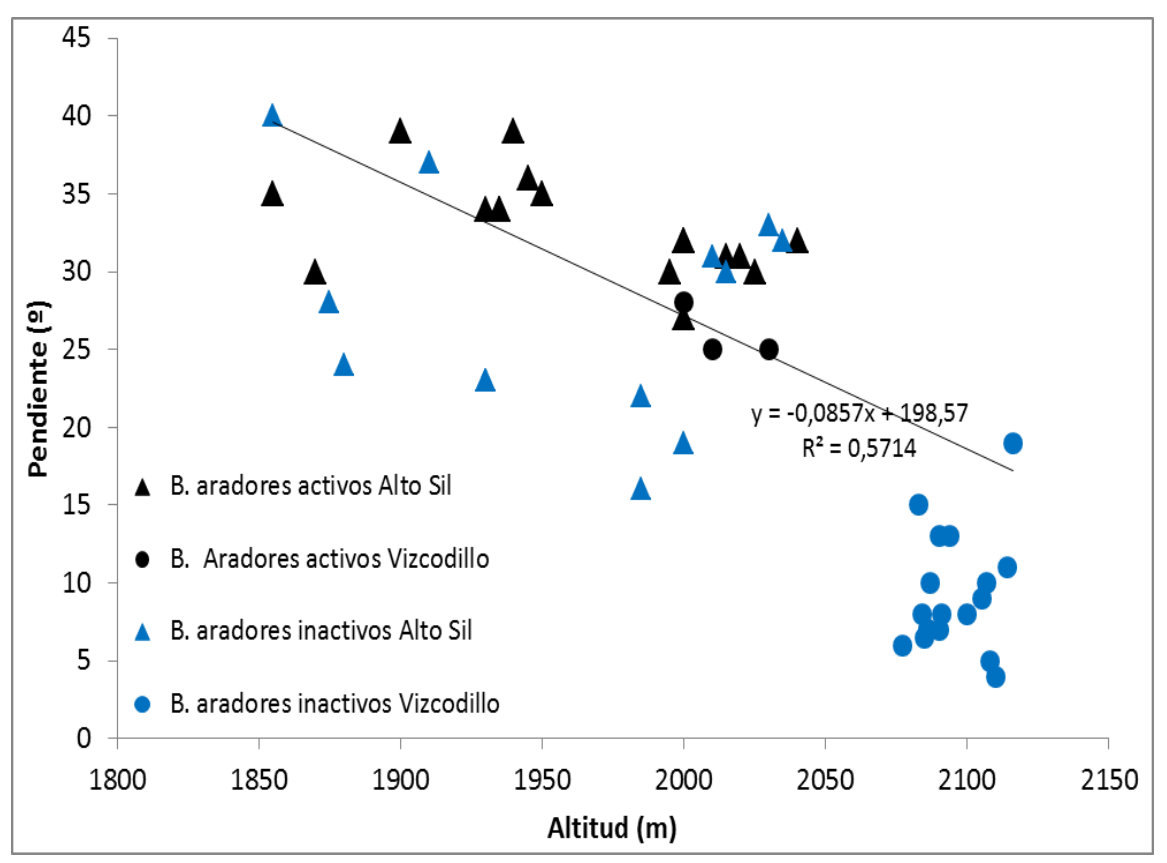

FUENTE: Elaboración de los autores

Figura 6. Relación entre la pendiente de la ladera donde se ubican los bloques aradores y la longitud del surco

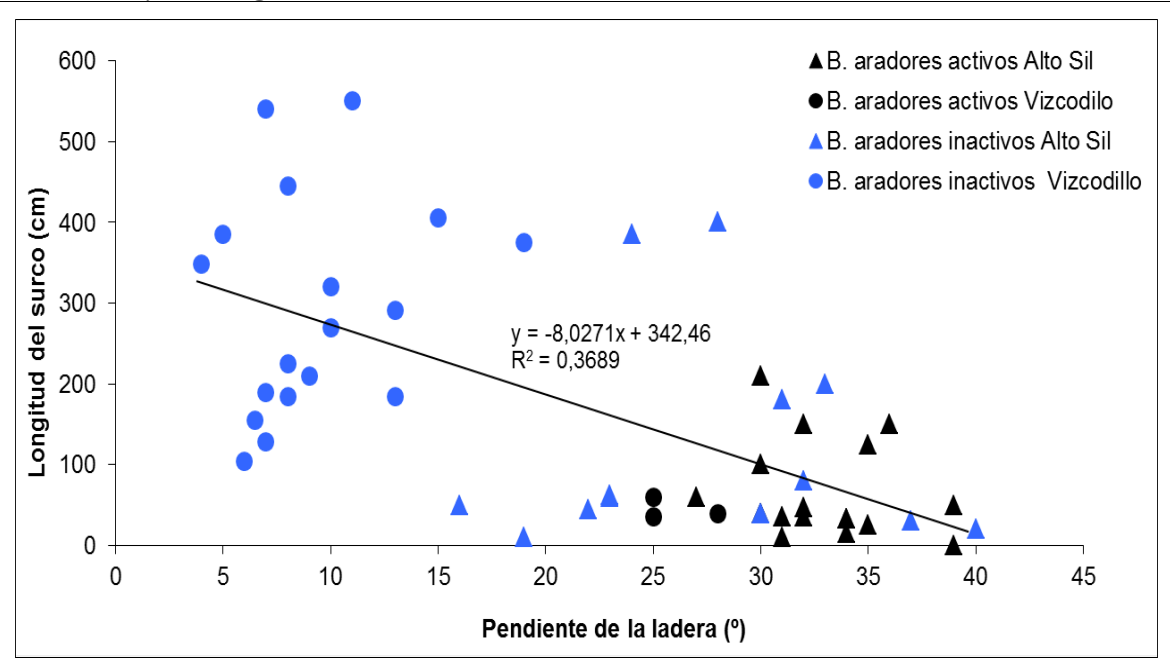

FUENTE: Elaboración de los autores 
Otra relación que puede ser significativa es la existente entre las dimensiones del montón frontal y el tamaño del surco, puesto que, en principio, a mayor material desplazado mayor debería ser también el material acumulado en el montón frontal. En la FIGURA 7 se observa que en los bloques inactivos el grado de correlación, aun no siendo excesivamente alto, es claro, y los montones tienden a ser más grandes en aquellos bloques que presentan un surco más largo, lo que indica que el material movilizado ha sido mayor. Sin embargo, en los bloques aradores activos el grado de correlación es muy bajo e incluso negativo.

Figura 7. Relación entre la longitud del surco y la altura del montón frontón

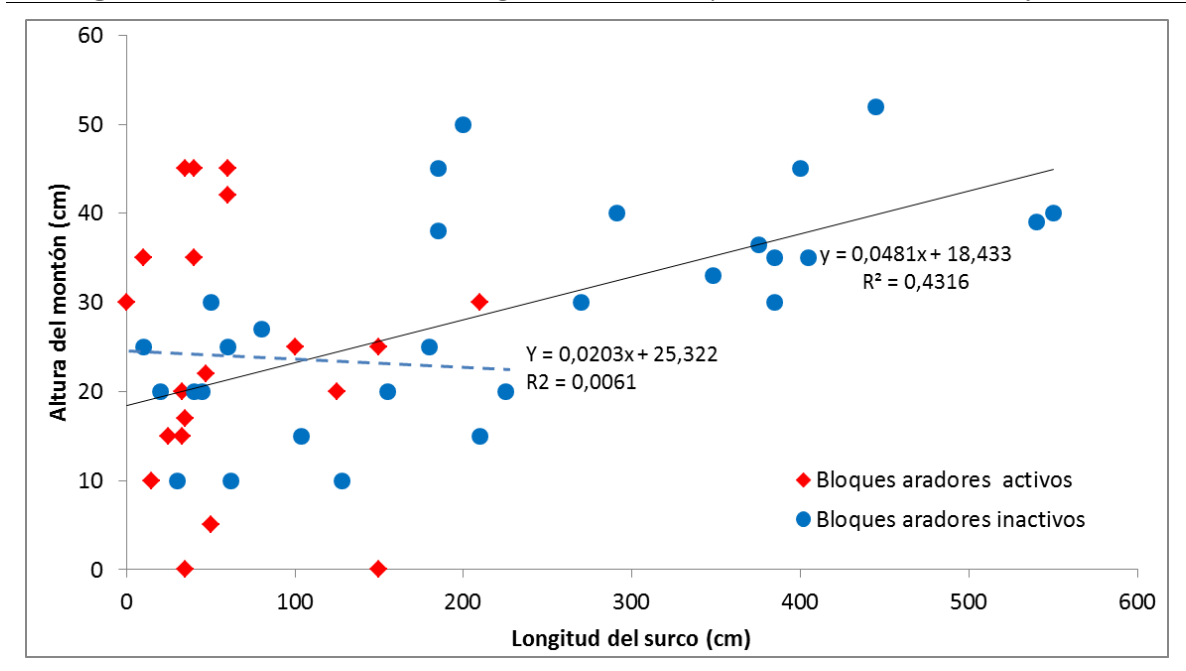

FUENTE: Elaboración de los autores

\section{IV.4. La relación de los bloques con el régimen térmico del suelo y la cubierta nival}

Los bloques aradores son una forma peculiar de gelifluxión, es decir, dependen de la congelación estacional del suelo para su formación, por lo que su aparición está ligada con ambientes donde las temperaturas medias son negativas al menos durante unos meses, o bien a zonas con permafrost. En la Península Ibérica eso solo ocurre en las zonas más elevadas de las principales cordilleras, por lo que son formas restringidas a algunas áreas de montaña.

Como ya dijimos en publicaciones anteriores (SANTOS GONZÁLEZ et al., 2009; SANTOS GONZÁLEZ, 2012), el régimen térmico del suelo en la zona culminante del Alto Sil está caracterizado por la congelación estacional del suelo en algunos emplazamientos, mientras que en otros la congelación está 
limitada a periodos breves de algunos días. En las solanas, las temperaturas raramente se mantienen por debajo de $0^{\circ} \mathrm{C}$ durante más de algunos días, con lo que la penetración del frente del hielo es muy escasa. En los neveros persistentes la congelación del suelo puede alcanzar varios meses, pero las temperaturas descienden solo muy ligeramente por debajo de los $0^{\circ} \mathrm{C}$, lo que impide una congelación profunda del suelo y fenómenos de gelifluxión. Sin embargo, las laderas con orientación norte o noroeste, en lugares donde la nieve tarda en establecerse durante el otoño, presentan localmente un régimen térmico del suelo más frío, con periodos de varios meses con temperaturas bajo $0^{\circ} \mathrm{C}$ y que pueden descender claramente de $\operatorname{los}-1^{\circ} \mathrm{C} \mathrm{o}-2^{\circ} \mathrm{C}$. En esas zonas la penetración del frente de helada puede alcanzar incluso unas pocas decenas de centímetros.

Figura 8. Régimen térmico del suelo de un bloque arador en el entorno de Valdeiglesia. Se pueden apreciar fusiones superficiales en varios periodos durante el invierno, mientras que a $-50 \mathrm{~cm}$ la congelación del suelo se produce a mediados de febrero.

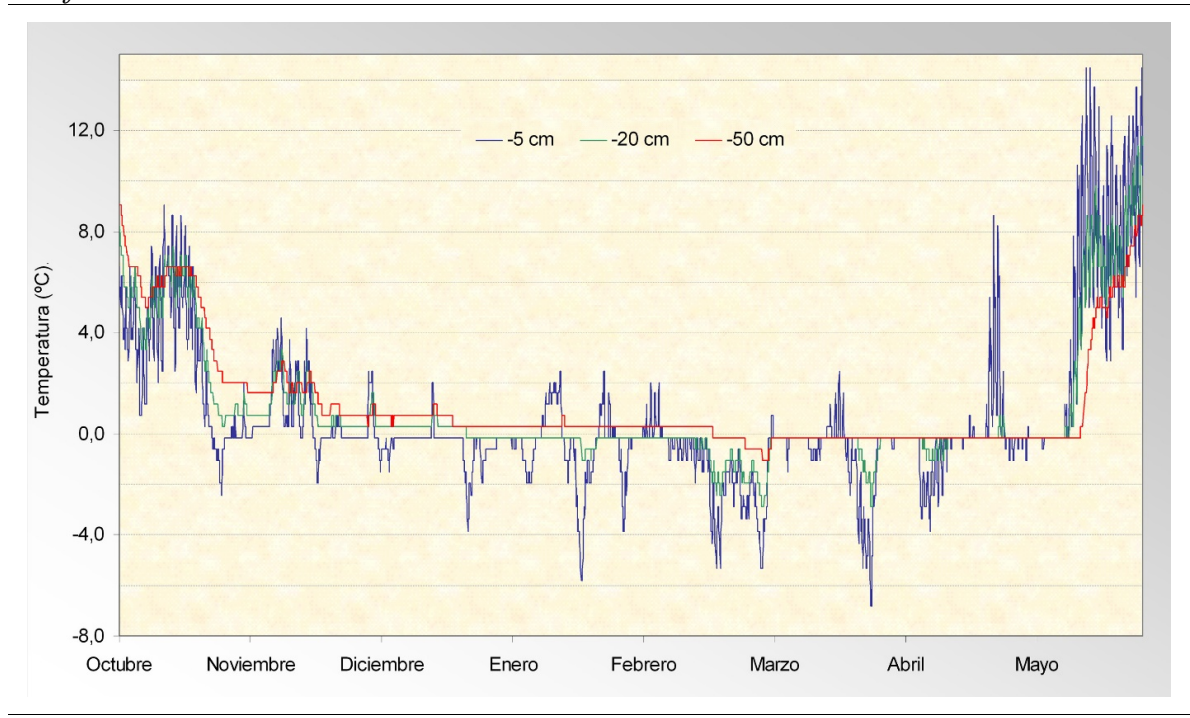

FUENTE: Elaboración de los autores

En particular, en un bloque arador en el entorno de Valdeiglesia, los datos obtenidos mostraron que la congelación del suelo podía alcanzar, como mínimo, los $50 \mathrm{~cm}$ (estimándose unos 70-80 cm), lo que sin duda favorece los procesos de gelifluxión (FIGURA 8). MATSUOKA (2010) también observa que son las zonas donde la cubierta nival es menos importante y reciben poca insolación térmica aquellas en las que congelación del suelo alcanza mayores profundidades. 
Todo ello lo que pone en evidencia es que los bloques se encuentran en lugares con unas condiciones térmicas favorables, estando muy condicionadas por la presencia de nieve. Así, los bloques se encuentran en zonas con una cubierta nival intensa, pero nunca dentro de zonas con neveros persistentes, es decir, la nieve ejerce un papel favorable por el aporte de agua al suelo, especialmente en el periodo de fusión, pero no se trata de formas relacionadas con la dinámica nival, sino con zonas con una congelación estacional profunda, y por tanto con una dinámica periglaciar. Estas observaciones están en consonancia con las realizadas por BALLANTYNE (2001: 270) y CARRERA GÓMEZ y PÉREZ ALBERTI (2006), que observan una cubierta de nieve importante pero menor que en otras zonas próximas donde hay neveros persistentes, si bien HALL et al. (2001: 220) los observa en zonas con una cubierta nival más persistente.

\section{DISCUSIÓN}

Los bloques aradores se forman únicamente en ambientes periglaciares, bien sea por la existencia de permafrost o de hielo estacional (TUFNELL, 1972; CHATTOPADHYAY, 1983; BERTHLING et al., 2001a; 2001b), si bien algunos autores consideran que, al menos en algunos casos, podrían estar asociados a procesos únicamente solifluidales (GARCÍA RUIZ et al., 1990; SERRANO CAÑADAS, 1998). De ahí que se puedan considerar indicadores de este tipo de ambientes, siempre que se pueda corroborar su actividad actual. En este sentido, aunque la mayor parte de los bloques aradores no presentan signos de desplazamientos actuales ni en los surcos ni en los montones, hay otros que sí se han mostrado activos en los periodos de observación, con lo que se pueden considerar indicadores climáticos que muestran la importancia de la dinámica periglaciar en las áreas de montaña analizadas.

Se considera que el movimiento de los bloques está relacionado con algunos factores como la formación de hielo de segregación en su entorno (GORBUNOV, 1991: 241; BALLANTYNE, 2001: 275) por su distinta conductividad térmica respecto al suelo adyacente (REID y NESJE, 1988) y la fusión del suelo más temprana en primavera, al calentarse el suelo más rápidamente por la presencia del propio bloque. En este sentido, GARCÍA DE CELIS et al. (2006) observan temperaturas más altas durante la tarde junto a un bloque, lo que puede provocar la fusión temprana del suelo de su entorno. Las fusiones invernales observadas en el bloque arador de Valdeiglesia pueden estar también relacionadas con el calentamiento del bloque.

Pero el movimiento también debe verse influenciado por la composición del suelo y sus características, sus límites plásticos y líquidos o su conductividad (BAllantyNe, 2001), que no han sido analizados en este trabajo. En todo 
caso, parece evidente que la presencia de los bloques se ve favorecida con suelos que tengan un porcentaje de material fino suficientemente elevado, como han señalado otros autores (WILSON, 1993; GARCÍA DE CELIS et al., 2006). Además, las causas que generan el movimiento no son del todo conocidas, como demuestra TUFNELL (1976) que observa mayores desplazamientos en los meses invernales. El aporte de agua de fusión nival, que llega a saturar la ladera, es un factor más que también parece ayudar al movimiento (SERRANO CAÑADAS, 1998; GRAB et al., 2008), siempre que se den otros factores como la congelación estacional profunda del suelo.

En el caso del Alto Sil y el macizo de Vizcodillo, los movimientos observados han sido muy evidentes debido a que provocan la movilización de sedimentos, que quedan desprovistos de vegetación, contrastando con el resto de las laderas en las que se ubican. Estos desplazamientos indican que la congelación del suelo alcanza profundidades relevantes, de algunas decenas de centímetros.

Pero la actividad de los bloques es, en primer lugar, ocasional y, en segundo, restringida a algunos de ellos. Es decir, por una parte solo se han observado bloques desplazados en años puntuales y no durante todos los años, lo que parece indicar que solo durante algunos años se alcanzan las condiciones térmicas favorables como para que se produzca el movimiento. Esas condiciones deben estar relacionadas tanto con la profundidad que alcance la congelación estacional del suelo, como a las condiciones en las que se produzca la fusión, lo que necesitaría estudios más detallados.

Por otra parte, algunos de los bloques son inactivos, mientras que otros muestran signos de actividad actual, aunque sea únicamente en años concretos. Como hemos visto, si analizamos la localización de los bloques inactivos y activos vemos algunas diferencias significativas. Así, los bloques aradores activos se encuentran casi siempre en zonas con pendiente más acusada y en ellos no se observa una correlación positiva entre la longitud del surco y la altura del montón frontal, al contrario de lo que ocurre en los inactivos. Esto puede indicar que los bloques aradores activos se encuentran en zonas más inestables, en las que la fuerte pendiente favorece por un lado el desplazamiento, como observó GORBUNOV (1991: 239), pero hace que los bloques puedan separarse del suelo y rotar, con lo que los avances son cortos y no se mantienen durante demasiados años. Por eso las longitudes de los surcos son menores en los activos. En este sentido, BALLANTYNE (2001: 278) señala que a partir de una pendiente límite (en torno a los $38-40^{\circ}$ ) pueden caer por gravedad y dejar por tanto de funcionar como bloques aradores. 
Por el contrario, muchos de los inactivos se encuentran en zonas de menor pendiente, donde los desplazamientos han sido más largos (como muestra la mayor longitud de los surcos), pero hoy en día no encuentran condiciones térmicas favorables para el movimiento. La densificación de la cubierta vegetal observada recientemente también debe ser un factor limitante para el movimiento de los bloques, si bien no se puede distinguir entre los efectos que haya podido tener un calentamiento del clima en la zona de una influencia del abandono de la ganadería.

También es significativo que los bloques activos se encuentran generalmente a menor altitud que los inactivos, ya que se ubican en zonas de la ladera donde aumenta la pendiente, y no en las partes culminantes, que presentan menores pendientes. Por tanto, aunque la altitud es relevante (no aparecen por debajo de los $1.850 \mathrm{~m}$ ), una vez alcanzado el valor mínimo el movimiento depende más de otras variables micro-climáticas.

El desplazamiento observado en los bloques ha sido de entre 10 y $35 \mathrm{~cm}$, valores elevados aunque en consonancia con los estimados por BALLANTYNE (2001: 278), que señala que el movimiento aumenta de forma exponencial en función de la pendiente, por lo que en el caso de los analizados la pendiente elevada explicaría por qué los movimientos observados son tan altos. En algunos bloques el desplazamiento era más difícil de medir con precisión y en otros se observaban expulsiones de material en la zona frontal similares a las observadas en otras zonas de la sierra de la Cabrera por CARRERA GóMEZ y PÉREZ ALBERTI (2006).

\section{CONCLUSIONES}

Una vez analizadas las características de medio centenar de bloques en el noroeste de la Península Ibérica, se han podido extraer una serie de conclusiones sobre los mismos. En particular, los bloques aradores solo aparecen en lugares con unas condiciones particulares como son:

- Altitudes superiores a los $1.850 \mathrm{~m}$.

- Laderas con orientación noroeste-sudeste, con pendientes muy variadas, entre $4^{\circ}$ y $40^{\circ}$.

— Laderas con materiales finos y bloques aislados que estén parcialmente dentro del suelo.

- Áreas con una cubierta nival persistente, pero fuera de los nichos de nivación, donde las temperaturas del suelo descienden menos. 
Todo lo anterior sirve para definir áreas con un régimen térmico que permita la congelación estacional del suelo de varios decímetros, donde se puedan producir fenómenos de gelifluxión. La nieve se comporta así como un agente que favorece el movimiento de los bloques por el aporte de agua con la fusión y el control del régimen térmico, pero no se puede hablar de formas nivales, sino periglaciares, asociadas a la congelación estacional (varios meses) del suelo, al menos en los casos analizados.

Es en esas zonas donde se encuentran los bloques aradores, que tienen unas dimensiones de entre 54 y $440 \mathrm{~cm}$ de eje mayor y están orientados preferentemente en el sentido de la ladera, en especial los bloques más alargados. Un $39 \%$ de los bloques presentan signos de movimientos actuales, si bien los desplazamientos se han producido únicamente en años concretos, lo que indica que se encuentran en unas condiciones límite. Además, los bloques activos se encuentran en zonas con pendientes elevadas, lo que favorece el movimiento.

Sin embargo, los bloques activos presentan surcos y montones frontales peor desarrollados y están peor alineados con la ladera que los inactivos, lo que nos lleva a interpretar que los desplazamientos han sido más reducidos, probablemente debido a que los bloques acaban despegándose del suelo y movilizándose por gravedad. Los valores de movimientos han sido relativamente altos, de hasta $35 \mathrm{~cm}$, probablemente también debido a que la pendiente ha favorecido tasas de desplazamiento más elevadas.

\section{BIBLIOGRAFÍA}

BALLANTYNE, C.K. (2001): «Measurement and theory of ploughing boulder movement». Permafrost and Periglacial Processes, ${ }^{\circ} 12$ (3), 267-288.

BERTHLING, I.; EIKEN, T.; MADSEN, H. y SOLLID, J.L. (2001a): «Downslope Displacement Rates of Ploughing Boulders in A Mid-Alpine Environment: Finse, Southern Norway». Geografiska Annaler, no 83A (3), 103-116.

BERTHLING, I.; EIKEN, T. y SOLLID, J.L. (2001b): «Frost heave and thaw consolidation of ploughing boulders in a mid-alpine environment, Finse, Southern Norway». Permafrost and Periglacial Processes, $\mathrm{n}^{\mathrm{o}} 12$ (2),165-177.

BRosCHE, K.U. (1978): «Formas actuales y límites inferiores periglaciares en la Península Ibérica». Estudios Geográficos, $\mathrm{n}^{\circ}$ 151, 131-161.

CARrera Gómez, P. y PÉrez ALBERTI, A. (2006): «Los bloques aradores de la vertiente septentrional del Cerro Fallaquinos. Montañas Galaico-Leonesas. Provincia de León. Rasgos morfométricos y mecanismos de movimiento», Póster en $I X$ Reunión Nacional de Geomorfología, (Santiago de Compostela, 13-15 septiembre de 2006),

Carrera Gómez, P.; Valcárcel díaz, M. y Pérez Alberti, A. (2005): «Ploughing blocks characteristics in the Faeda Cirque, northwestern Spain». Póeter en F. Gutiérrez, M. Gutiérrez, G. Desir, J. Guerrero, P. Lucha, C. 
MARÍN y J.M. GARCÍA RUIZ (Eds.), Sixth International Conference on Geomorphology, Abstracts volume, Zaragoza, pp. 20.

ChatTopadHYAY, G.P. (1983): «Ploughing blocks on the Drumochter Hills in the Grampian Highlands, Scotland: a quantitative report». The Geographical Journal, $n^{\circ} 149$ (2), 211-215.

Del BARrio, G. y Puigdefábregas, J. (1987): «Mass wasting features above the timberline in the Central Pyrenees and their topographic control». Pirineos, $\mathrm{n}^{\circ} 130$, 29-51.

DiONNE, J.C. (1988): «Ploughing boulders around shorelines, with particular reference to the St. Lawrence estuary». Geomorphology, n ${ }^{\circ} 1$ (4), 297-308.

FRÄNZLE, O. (1959): «Glaziale und periglaziale Formbildung im östlichen Kastilischen Scheidegebirge (Zentralspanien)». Bonner Geographische Abhandlungen, $n^{\circ} 26,1-80$.

FRÄNZLE, O. (1978): «Formaciones glaciares y periglaciares en el sector oriental del Sistema Central». Estudios Geográficos, nº 151, 203-231 y nº152, 363-418.

FRENCH, H.M. (1996): The periglacial environmet. Singapore, Longman.

García De Celis, A.; Arroyo PÉrez, P. y Gandía Fernández, A. (2006): «Los bloques aradores de "Laguna Helada" (Urbión): un ejemplo singular de solifluxión activa en la Cordillera Ibérica». Polígonos. Revista de Geografía, n 16, 119-132.

García-Ruiz, J.M., Alvera, B., Del Barrio, G. y Puidefábregas, J., (1990): «Geomorphic processes above timberline in the Spanish Pyrenees». Mountain Research and Development, 10 (3): 201-214.

GÓMEZ-VILlAR, A.; GONZÁLEZ-GuTIÉRREZ, R.B.; REDONDO-VEGA, J.M. y SANTOSGONZÁLEZ, J. (2011): «Distribución de glaciares rocosos relictos en la Cordillera Cantábrica». Cuadernos de Investigación Geográfica, 37 (2), 49-80. DOI: http://dx.doi.org/10.18172/cig.1256

GÓMEZ-VILlar, A.; SANTOS-GONZÁleZ, J.; GONZÁlEZ-GutiÉRREZ, R.B. y REDONDO-VEGA, J.M. (2015): "Glacial cirques in the southern side of the Cantabrian Mountains of southwestern Europe». Geografiska Annaler, no 97 (4), 633651. DOI: 10.1111 /geoa.12104

GONZÁLEZ GUTIÉRREZ, R.B. (2001): Estudio geomorfológico de la Montaña Central de León: los valles del Torío y Curueño. Tesis doctoral (inédita). Departamento de Geografía, Universidad de León, 466 pp.

GonZÁlez GutiÉRrez, R.B. (2002): El relieve de los valles del Torío y Curueño (Montaña Cantábrica Leonesa). León, Universidad de León.

GorbunOV, A.P. (1991): «Ploughing blocks of the Tien Shan». Permafrost and Periglacial Processes, $\mathrm{n}^{\circ} 2$ (3), 237-243.

GRAB, S.W.; DiCKINSON, K.J.M. y MARK, A.F. (2008): «Ploughing boulders on the Rock and Pillar Range, south-central New Zealand: their geomorphology and alpine plant associations». Journal of the Royal Society of New Zealand, $\mathrm{n}^{\mathrm{o}} 38$ (1), 51-70.

GutiÉRrez ElorZA, M. y PEÑA Monné, J.L. (1981): «Los glaciares rocosos y el modelado acompañante en el área de la Bonaigua (Pirineo de Lérida)». Boletín Geológico y Minero, no 92, 11-20. 
HALl, K.; BoelhouWers, J. y DRISCOLL, K. (2001): «Some morphometric measurements on ploughing blocks in the McGregor Mountains, Canadian Rockies». Permafrost and Periglacial Processes, $\mathrm{n}^{\circ} 12$ (2), 219-225.

HreŠKO, J., BUGÁr, G., BOLTIŽIAR, M. y KoHÚT, F. (2008): «The dynamics of recent geomorphic processes in the alpine zone of the Tatra Mountains» en Geographia Polonica, 81 (1). (RĄCZKOWSKA, Z. y STARKEL, L. eds.). Warszawa, Modelling of high-mountain relief. Polish Academy of Sciences, 53-65.

MATSUOKA, N. (2010): «Solifluction and mudflow on a limestone periglacial slope in the Swiss Alps: 14 years of monitoring». Permafrost and Periglacial Processes, 21 (3): 219-240.

Oliva, M.; Schulte, L. y Gómez OrTiz, A. (2009): «Morphometry and Late Holocene activity of solifluction landforms in the Sierra Nevada, southern Spain». Permafrost and Periglacial Processes, $\mathrm{n}^{\mathrm{o}}$ 20, 369-382.

Pérez Alberti, A., Valcárcel díAz, M., Martínez Cortizas, A. y Blanco CHAO, R., (1998): «Evidencias de procesos periglaciares actuales en el noroeste de la Península Ibérica» en Procesos biofísicos actuales en medios fríos (GóMEZ Ortiz, A.; Salvador Franch, F.; Schulte L. y García Navarro A., eds.). Barcelona, Publiacions de la Universitat de Barcelona, 245-261.

REdondo Vega, J.M., Gómez Villar, A., GonzÁlez GutiÉrrez, R.B. y SANTOS GonzÁlez, J. (2010): Los glaciares rocosos de la Cordillera Cantábrica. León, Universidad de León.

Redondo Vega, J.M., Gómez Villar, A., GonZÁlez GutiÉrRez, R.B. y CARrera GómEZ, P. (2002): «El relieve glaciar del macizo de Vizcodillo, Cabrera Alta, León» en El modelado de origen glaciar en las montañas leonesas (REDONDO Vega, J.M.; Gómez Villar A.; GonzÁlez GutiÉrrez R.B. y P. CARRERA GÓMEZ, coords.), León, Universidad de León, 13-25.

REID, J.R. y NESJE, A. (1988): «A giant ploughing block, Finse, southern Norway». Geografiska Annaler, no 70A (1-2), 27-33.

SANJosÉ BlAsco, J.J., SERRANO CAÑADAS, E. y GóMEZ LENDE, M. (2015): «Análisis geomático de bloques aradores y lóbulos en los Puertos de Áliva (Picos de Europa, Cordillera Cantábrica)»V Congreso Ibérico de la I.P.A., Valladolid.

SANTOS GonZÁLEZ, J. (2012): Glaciarismo y periglaciarismo en el Alto Sil, provincia de León (Cordillera Cantábrica). León, Universidad de León (DVD).

SANTOS GONZÁlEZ, J.; GONZÁlEZ GuTIÉRREZ, R.B.; GÓMEZ VILlAR, A. y REDONDO VEGA, J.M. (2009): «Ground thermal regime in the vicinity of fossil rock glaciers (Cantabrian Mountains, NW Spain)». Finisterra, n $^{\circ} 87,35-44$.

SAntos GonzÁlez, J.; Redondo Vega, J.M.; Gómez Villar, A. y GonzÁlez GUTIÉRREZ, R.B. (2010): «Dinámica actual de los nichos de nivación del Alto Sil (Cordillera Cantábrica)». Cuadernos de Investigación Geográfica, 36 (1): 87-106.

SANTOS-GonZÁleZ, J.; REDONDO-VEGA, J.M.; GONZÁlEZ-GuTIÉRREZ, R.B. y GÓMEZ-VILLAR, A. (2013): «Applying the AABR method to reconstruct equilibrium-line altitudes from the last glacial maximum in the Cantabrian Mountains (SW Europe)». Palaeogeography, Palaeoclimatology, Palaeoecology, $\mathrm{n}^{\circ} 387$, 185-199. doi: 10.1016/j.palaeo.2013.07.025 
SERrano CaÑADAS, E. y GonZÁlez TruebA, J.J. (2004): «Morfodinámica periglaciar en el grupo Peña Vieja (Macizo Central de los Picos de Europa -Cantabria-)». Cuaternario y Geomorfología, $\mathrm{n}^{\mathrm{o}} 18$ (3-4), 73-88.

SERRANO CAÑADAS, E. (1995): «Geomorfología de la Sierra de Tendeñera (Pirineo aragonés)». Ería, no 37, 143-158.

SERRANO CAÑADAS, E., 1998. Geomorfología del Alto Gállego (Pirineo aragonés). Institución «Fernando el Católico», Zaragoza, $501 \mathrm{pp}$.

TUFNELL, L. (1972): «Ploughing blocks with special reference to north-west England». Biuletyn Peryglacjalny, $\mathrm{n}^{\mathrm{o}}$ 21, 237-270.

TUFNELL, L. (1976): «Ploughing block movements on the Moor House Reserve (England), 1965-75». Biuletyn Periglacjalny, $\mathrm{n}^{\circ}$ 26, 311-317.

VAlcárcel Díaz, M.; Blanco ChaO, R.; Martínez Cortizas, A. y PÉrez AlBERTI, A. (1998): «Estimación de las paleotemperaturas en Galicia durante el último ciclo glaciar a partir de datos geomorfológicos y climáticos» en Investigaciones recientes de la geomorfología española (GÓMEZ ORTIZ, A. y SALVADOR FRANCH F. eds.), Servei de Paisatge-UB, Huesca, Geoforma Ediciones, 767-770.

WASHBURN, A.L. (1973): Periglacial processes and environments. London, Arnolds Publications.

WILSON, P. (1993): «Ploughing-boulder characteristics and associated soil properties in the Lake District and southern Scotland». Scottish Geographical Magazine, $\mathrm{n}^{\circ}$ 109 (1), 18-26.

\section{ANEXO 1. CARACTERÍSTICAS MORFOMÉTRICAS DE LOS BLO- QUES ARADORES DEL ALTO SIL (AS) Y VIZCODILLO (VI)}

\begin{tabular}{|c|c|c|c|c|c|c|c|c|c|c|c|}
\hline Zona & Alt & $L$ & $A$ & $A$ & $P l$ & $\mathrm{Ol}$ & $\mathrm{Ob}$ & $L s$ & $\mathrm{Am}$ & $T m$ & Din \\
\hline AS & 2040 & 169 & 165 & 80 & 32 & 23 & 27 & 150 & 25 & Frontal & Sí \\
\hline AS & 2035 & 89 & 65 & 30 & 32 & 23 & 42 & 80 & 27 & Frontal & \\
\hline AS & 2030 & 133 & 108 & 33 & 33 & 37 & 71 & 200 & 50 & F.lat. & \\
\hline AS & 2025 & 115 & 104 & 22 & 30 & 15 & 110 & 100 & 25 & Frontal & Sí \\
\hline AS & 2020 & 91 & 68 & 17 & 31 & 25 & 120 & 10 & 35 & Frontal & Sí \\
\hline AS & 2015 & 91 & 54 & 15 & 30 & 22 & 28 & 40 & 20 & Frontal & \\
\hline AS & 2015 & 59 & 41 & 21 & 31 & 24 & 45 & 35 & 0 & Later. & Sí \\
\hline AS & 2010 & 178 & 77 & 63 & 31 & 15 & 37 & 180 & 25 & F.lat. & \\
\hline AS & 2000 & 126 & 45 & 20 & 32 & 72 & 85 & 35 & 17 & Frontal & Sí \\
\hline AS & 2000 & 116 & 83 & 20 & 32 & 72 & 70 & 47 & 22 & Frontal & Sí \\
\hline AS & 2000 & 258 & 102 & 39 & 19 & 106 & 110 & 10 & 25 & Frontal & \\
\hline AS & 2000 & 250 & 178 & 32 & 27 & 106 & 106 & 60 & 45 & Frontal & Sí \\
\hline AS & 1995 & 120 & 100 & 38 & 30 & 103 & 103 & 210 & 30 & F.lat. & Sí \\
\hline AS & 1985 & 127 & 68 & 22 & 22 & 85 & 28 & 45 & 20 & Frontal & \\
\hline AS & 1985 & 143 & 118 & 20 & 16 & 68 & 155 & 50 & 30 & F.lat. & \\
\hline AS & 1950 & 134 & 61 & 53 & 35 & 354 & 345 & 125 & 20 & F.lat & Sí \\
\hline AS & 1945 & 67 & 32 & 16 & 36 & 5 & 15 & 150 & 0 & Frontal & Sí \\
\hline AS & 1940 & 94 & 86 & 15 & 39 & 10 & 265 & 0 & 30 & F.lat & Sí \\
\hline AS & 1935 & 77 & 65 & 36 & 34 & 0 & 315 & 33 & 15 & Frontal & Sí \\
\hline AS & 1935 & 105 & 38 & 27 & 34 & 345 & 343 & 15 & 10 & Lateral & Sí \\
\hline AS & 1930 & 90 & 86 & 31 & 34 & 356 & 95 & 33 & 20 & Frontal & Sí \\
\hline AS & 1930 & 158 & 87 & 49 & 23 & 355 & 73 & 60 & 25 & Frontal & \\
\hline AS & 1930 & 153 & 45 & 20 & 23 & 8 & 8 & 62 & 10 & F.lat & \\
\hline AS & 1910 & 54 & 38 & 23 & 37 & 1 & 1 & 30 & 10 & F.lat & \\
\hline AS & 1900 & 79 & 33 & 29 & 39 & 5 & 287 & 50 & 5 & F.lat & Sí \\
\hline AS & 1880 & 146 & 144 & 85 & 24 & 80 & 135 & 385 & 35 & F.lat & \\
\hline AS & 1875 & 192 & 150 & 55 & 28 & 357 & 15 & 400 & 45 & F.lat & \\
\hline
\end{tabular}




\begin{tabular}{|c|c|c|c|c|c|c|c|c|c|c|c|}
\hline Zona & Alt & $L$ & $A$ & $A$ & $P l$ & $\mathrm{Ol}$ & $O b$ & $L s$ & $\mathrm{Am}$ & $\mathrm{Tm}$ & Din \\
\hline AS & 1870 & 125 & 65 & 35 & 30 & 352 & 320 & 40 & 35 & Frontal & Sí \\
\hline AS & 1855 & 110 & 46 & 19 & 35 & 350 & 353 & 25 & 15 & Frontal & Sí \\
\hline AS & 1855 & 73 & 52 & 24 & 40 & 353 & 65 & 20 & 20 & Frontal & \\
\hline VI & 2116 & 332 & 132 & 66 & 19 & 332 & 299 & 375 & 36,5 & Frontal & \\
\hline VI & 2114 & 335 & 230 & 126 & 11 & 28 & 33 & 550 & 40 & Frontal & \\
\hline VI & 2110 & 307 & 93 & 63 & 4 & 96 & 105 & 348 & 33 & F.lat & \\
\hline VI & 2108 & 320 & 191 & 95 & 5 & 97 & 114 & 385 & 30 & F.lat & \\
\hline VI & 2107 & 400 & 283 & 115 & 10 & 95 & 253 & 320 & 75 & F.lat & \\
\hline VI & 2105 & 260 & 242 & 110 & 9 & 96 & 118 & 210 & 15 & F.lat & \\
\hline VI & 2100 & 256 & 225 & 59 & 8 & 97 & 268 & 225 & 20 & Frontal & \\
\hline VI & 2094 & 272 & 151 & 55 & 13 & 102 & 94 & 291 & 40 & Frontal & \\
\hline VI & 2091 & 219 & 128 & 77 & 8 & 75 & 167 & 185 & 45 & Frontal & \\
\hline VI & 2090 & 140 & 69 & 52 & 13 & 136 & 322 & 185 & 38 & Frontal & \\
\hline VI & 2090 & 197 & 160 & 102 & 7 & 96 & 98 & 540 & 39 & F.lat & \\
\hline VI & 2087 & 250 & 137 & 59 & 10 & 15 & 215 & 270 & 30 & Frontal & \\
\hline VI & 2086 & 423 & 305 & 55 & 7 & 81 & 297 & 190 & 102 & Frontal & \\
\hline VI & 2086 & 208 & 158 & 75 & 7 & 106 & 304 & 128 & 10 & Lateral & \\
\hline VI & 2085 & 338 & 149 & 116 & 6,5 & 114 & 326 & 155 & 20 & Frontal & \\
\hline VI & 2084 & 340 & 129 & 57 & 8 & 104 & 305 & 445 & 52 & Frontal & \\
\hline VI & 2083 & 440 & 280 & 69 & 15 & 358 & 6 & 405 & 35 & F.lat & \\
\hline VI & 2077 & 310 & 85 & 83 & 6 & 9 & 32 & 104 & 15 & Frontal & \\
\hline VI & 2030 & 335 & 240 & 70 & 25 & 350 & 5 & 60 & 42 & Frontal & Sí \\
\hline VI & 2010 & 145 & 88 & 56 & 25 & 360 & 10 & 35 & 45 & Frontal & Sí \\
\hline VI & 2000 & 140 & 120 & 60 & 28 & 355 & 10 & 40 & 45 & Frontal & Sí \\
\hline
\end{tabular}

Alt (Altitud). L (Longitud, cm.). A (Anchura, cm). a (Altura del bloque, $\mathrm{cm}$ ). Pl (Pendiente ${ }^{\circ}$ ) de la ladera). Ol (Orientación de ladera). Ob (Orientación del bloque). Ls (Longitud del surco, cm). Am (Altura del montón, cm). Tm (Tipo de montón). Din (Dinámica actual). 